\title{
Treatment profiles and trajectories surrounding the diagnosis of major depressive disorder: a research protocol for a Danish register-based study. [version 1; peer review: awaiting peer
} review]

\author{
Pernille Herold Jeberg (D1,2, Anne Marije Christina Overgaard Nielsen (D1,2, \\ Merete Osler1,3, Marie Kim Wirum-Andersen', Ramune Jacobsen (iD2, \\ Anna Birna Almarsdóttir (iD2, Kristoffer Jarlov Jensen1, Janne Petersen ${ }^{1,4}$ \\ ${ }^{1}$ Center for Clinical Research and Prevention, Bispebjerg and Frederiksberg Hospital, Copenhagen, Denmark \\ 2Section of Social and Clinical Pharmacy, Department of Pharmacy, University of Copenhagen, Copenhagen, Denmark \\ ${ }^{3}$ Section of Epidemiology, Department of Public Health, University of Copenhagen, Copenhagen, Denmark \\ ${ }^{4}$ Section of Biostatistics, Department of Public Health, University of Copenhagen, Copenhagen, Denmark
}

V1 First published: 16 Feb 2022, 11:193
https://doi.org/10.12688/f1000research.75010.1

Latest published: 16 Feb 2022, 11:193

https://doi.org/10.12688/f1000research.75010.1

\section{Open Peer Review}

Approval Status AWAITING PEER REVIEW

Any reports and responses or comments on the article can be found at the end of the article.

\section{Abstract}

Background: Major depressive disorder (MDD) is a prevalent illness that causes significant suffering and expenses at the personal and societal levels. The disorder is subject to heterogeneity reflected by diverse clinical phenotypes and assorted responses to treatment. Research on MDD treatments have focused on one treatment at a time, however many patients receive several different treatments. Considering the number of available treatment options, we hypothesize that it is possible to identify clinically meaningful groups of patients based on their psychiatric treatment. The objective of this study is therefore to identify psychiatric treatment profiles and trajectories of patients with major depressive disorder and, for the identified profiles and trajectories, to assess clinical and sociodemographic factors.

Method: The study will be a population-based register study of patients with major depressive disorder in the Danish National Patient Register between 2011 and 2015. Using latent class analyses, we will identify homogenous groups of patients based on their psychiatric treatment patterns. These patterns constitute psychiatric treatment profiles which will be identified at six time-intervals, from 1.5 years before to 3 years after diagnosis of major depressive disorder. By cross-tabulating the identified treatment profiles, we will establish psychiatric treatment trajectories. Patients sharing profiles and trajectories will be characterized.

Discussion: Identification of psychiatric treatment profiles and 
trajectories based on an unsupervised learning algorithm have the potential to reveal hidden patterns of psychiatric treatment. This will potentially pave the way for future studies of treatment combinations and a larger insight into the different courses of treatment.

Furthermore, the assessment of clinical and sociodemographic factors may indicate different patient characteristics across treatment profiles and trajectories.

\section{Keywords}

Denmark, Depression, latent class analysis, treatment profile, treatment trajectory.

\section{Corresponding author: Pernille Herold Jeberg (pernille.herold.jeberg@regionh.dk)}

Author roles: Herold Jeberg P: Conceptualization, Methodology, Project Administration, Visualization, Writing - Original Draft Preparation, Writing - Review \& Editing; Overgaard Nielsen AMC: Conceptualization, Methodology, Writing - Review \& Editing; Osler M: Conceptualization, Writing - Review \& Editing; Kim Wirum-Andersen M: Conceptualization, Writing - Review \& Editing; Jacobsen R: Conceptualization, Writing - Review \& Editing; Almarsdóttir AB: Conceptualization, Supervision, Writing - Review \& Editing; Jarlov Jensen K: Conceptualization, Methodology, Supervision, Writing - Review \& Editing; Petersen J: Conceptualization, Methodology, Supervision, Writing - Review \& Editing

Competing interests: No competing interests were disclosed.

Grant information: The author(s) declared that no grants were involved in supporting this work.

Copyright: @ 2022 Herold Jeberg P et al. This is an open access article distributed under the terms of the Creative Commons Attribution License, which permits unrestricted use, distribution, and reproduction in any medium, provided the original work is properly cited.

How to cite this article: Herold Jeberg P, Overgaard Nielsen AMC, Osler M et al. Treatment profiles and trajectories surrounding the diagnosis of major depressive disorder: a research protocol for a Danish register-based study. [version 1; peer review: awaiting peer review] F1000Research 2022, 11:193 https://doi.org/10.12688/f1000research.75010.1

First published: 16 Feb 2022, 11:193 https://doi.org/10.12688/f1000research.75010.1 


\section{Introduction}

Major depressive disorder (MDD) has considerable personal and societal consequences, and 15-18\% of all individuals will suffer from MDD in their lifetime. ${ }^{1-3}$ The phenotypic expression characterizes the disorder by a depressed mood, reduced energy and/or loss of interest and enjoyment. ${ }^{4,5}$ Despite many years of research, the aetiology and pathophysiology of MDD have not been fully clarified. ${ }^{6,7}$ The disorder is considered heterogenous, reflected by diverse clinical phenotypes, inconsistent biological measures, and assorted and suboptimal responses to treatment. ${ }^{5-10}$ The available treatments fail to treat all patients successfully; $30 \%$ are characterized as treatment-resistant, and even more have a suboptimal treatment outcome. ${ }^{10}$

Gronemann et al. found that the rate of treatment-resistant depression is higher for patients redeeming specific psychotropic co-medicines (e.g. benzodiazepines), suggesting that certain MDD patients have fewer shifts in treatment as comorbid somatic disorders may restrict treatment options due to drug-drug interactions. ${ }^{11}$ Considering the number of available treatment options, we hypothesize that it is possible to identify clinically meaningful groups of MDD patients based on their psychiatric treatment. Psychiatric MDD treatments include an array of pharmacological and nonpharmacological treatments, and combinations thereof, and is characterized by multiple shifts in treatment over time. ${ }^{12}$ However, most literature is restricted to studying one treatment at a time instead of treatment profiles. The present study uses a multivariate method (latent class analysis), suitable for identifying data-driven homogenous groups of patients based on a set of information. Here, these groups represent psychiatric treatment profiles based on redeemed prescriptions and non-pharmacological treatments. No studies have previously grouped MDD patients based on their psychiatric treatment by using latent class analysis, nor used psychiatric treatment profiles to investigate treatment trajectories.

The objectives of this study are 1) to identify treatment profiles based on patients' psychiatric treatment received during six time intervals positioned before and after depression diagnosis, 2) to link treatment profiles into trajectories over at least three intervals, and 3) to describe patients with similar treatment profiles and trajectories in relation to clinical and sociodemographic characteristics.

\section{Protocol}

Study design and settings

The present study is a register-based study of patients with a first-time hospital contact for MDD (index date), identified in the Danish National Patient Registry (DNPR) from January $1^{\text {st }}, 2011$ through December $31^{\text {st }}$, 2015. Six four-month intervals (Interval 1-6) will be arranged relative to the index date, corresponding to the date of diagnosis; -18 to $-14,-4$ to 0,0 to 4,4 to 8,18 to 22 and 32 to 36 months (Figure 1 ).

\section{Population}

The study population is a cohort of patients registered at a psychiatric unit, with MDD (International Classification of Diseases $10^{\text {th }}$ revision [ICD-10]): F32 [single episode] or F33 [recurrent]) as a primary diagnosis from 2011 to 2015.

Patients will not be eligible if they 1) had a primary or secondary diagnosis of MDD, dementia (F00-F03) in a period of 15 years prior to index date, schizophrenia (F20), manic episodes (F30) or bipolar depression (F31); 2) if they migrated out of/into Denmark in the same period; 3 ) if their date of birth was not registered within the Danish Civil Registration System (CRS) for the period 2011-2015; or 4) were younger than 10 years of age at index date (Figure 2).

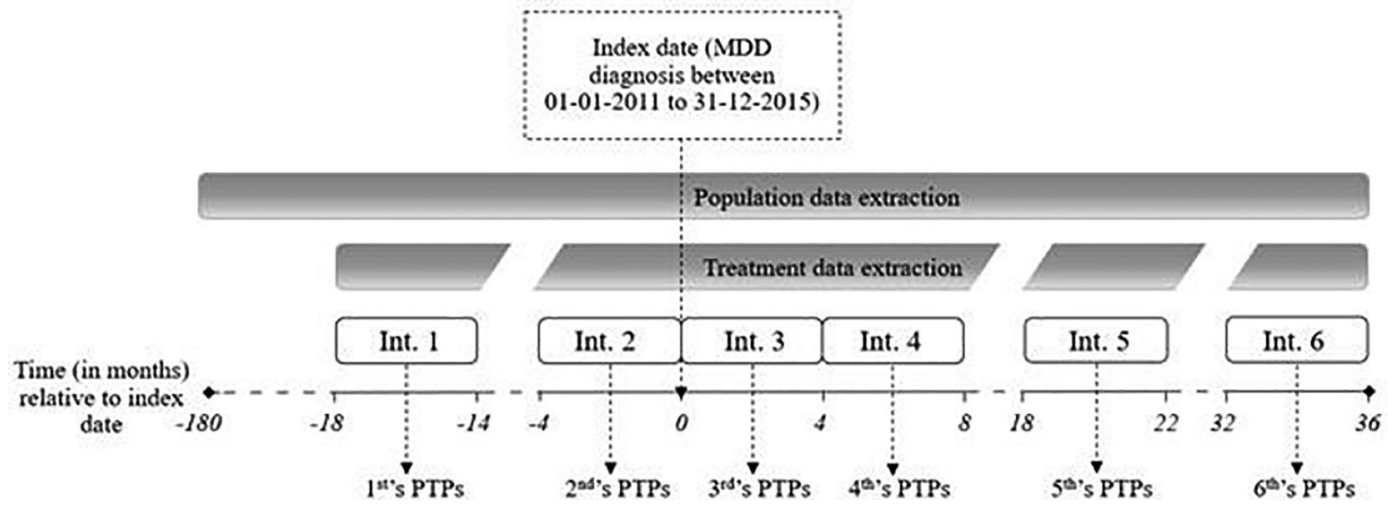

Figure 1. Timeline relative to index date with Interval 1-6 positioned prior to and past index date. Grey bars indicate data extraction; the "population data extraction" bar is data necessary to establish the cohort, sub-cohorts and descriptive variables, whereas the "treatment data extraction" bar indicates data required for psychiatric treatment profile (PTP) modelling. 


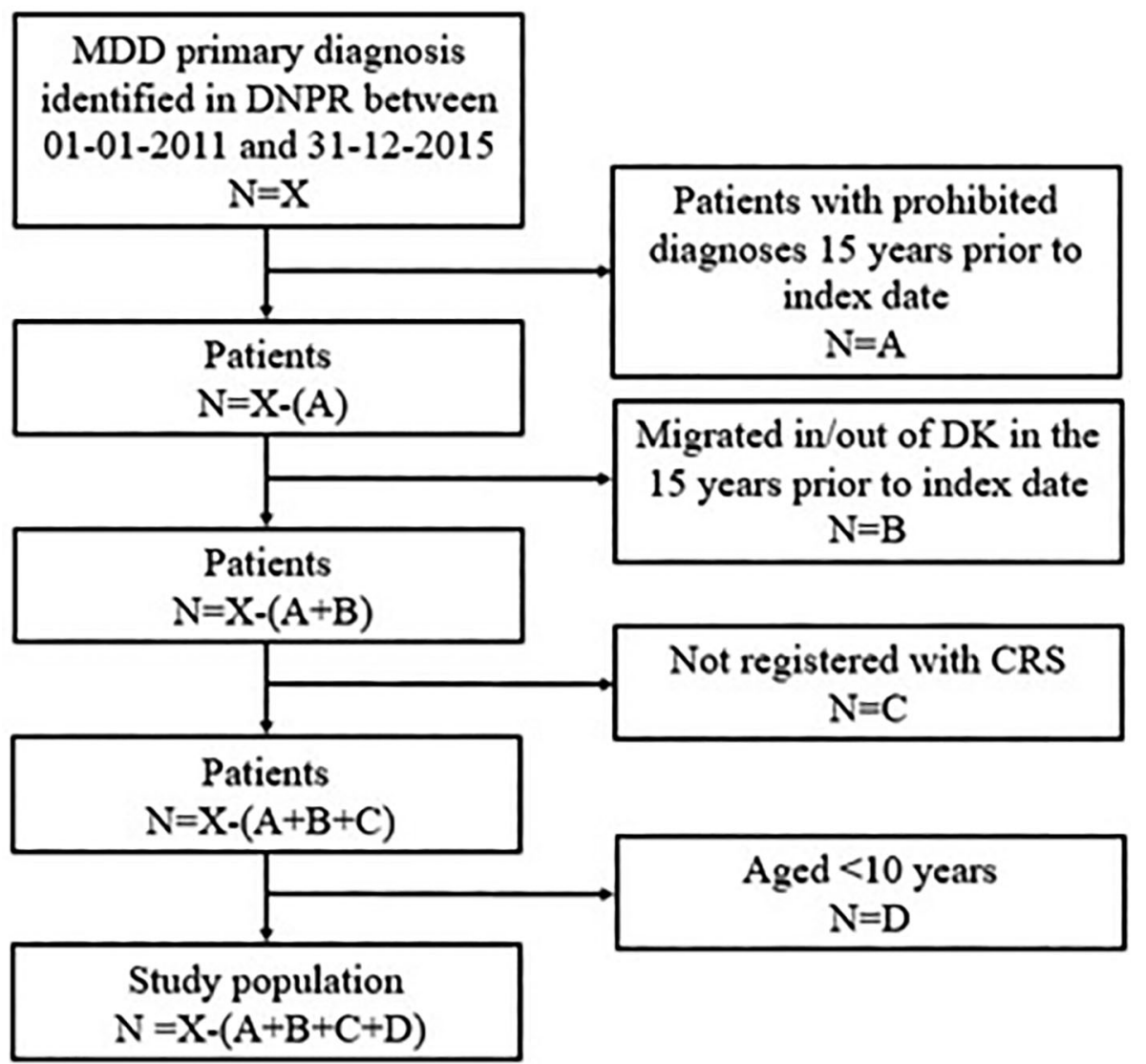

Figure 2. Flowchart of inclusion and exclusion criteria. Patients diagnosed with major depressive disorder (MDD) as primary diagnosis between 01-01-2011 and 31-12-2015 will be included. Patients will be excluded if they A) were diagnosed with MDD, dementia, schizophrenia, manic episodes or bipolar depression 15 years prior to index date, B) migrated in or out of Denmark 15 years prior to index date, C) had incomplete data portfolios or D) were aged under 10 years of age at the time of diagnosis.

Interval-specific sub-cohorts will be established based on a follow-up assessment applied past the index date, i.e. at Interval 3-6. Patients will be excluded if they i) migrated out of/into Denmark throughout an interval, ii) migrated out of Denmark, between two intervals, and did not return before the interval began or iii) died before or within an interval (Figure 3).

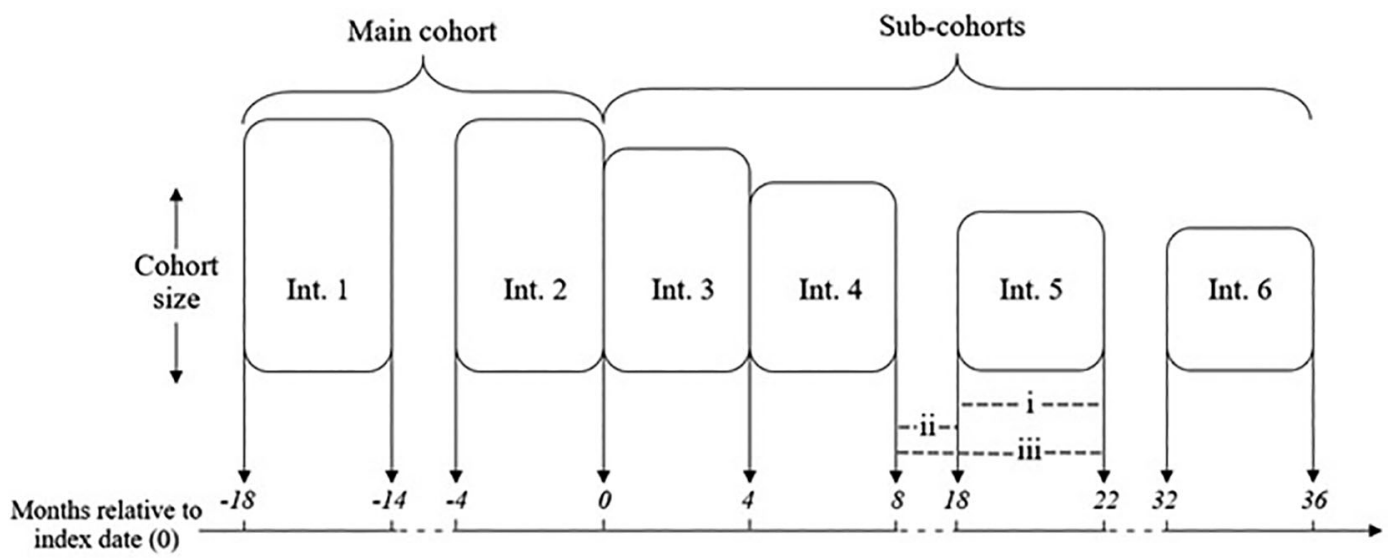

Figure 3. Follow-up assessment; interval specific sub-cohorts will be established for intervals past index date (time 0). Establishment of the sub-cohort of Interval 5 is used as an example. Patients will be excluded if they i) migrated within an interval, ii) migrated out of Denmark without returning before the beginning of the interval, or iii) died between or within intervals. 
Data sources and variables

The Danish health care system provides universal coverage and is largely tax-funded. ${ }^{13,14}$ Data on the Danish population are, mainly for administrative purposes, reported into nationwide registers and cross-linkage at the individual level is enabled by a unique personal identification number. ${ }^{13-15}$ The CRS dates back to 1968 and includes data on date of birth, sex, vital status, migration, among others. ${ }^{16-18}$ The DNPR dates back to 1977 and includes data on in- and outpatients from hospitals. Data includes variables regarding diagnostics and treatment. ${ }^{15,17,19,20}$ The Danish National Prescription Register (NPR) dates back to 1994 and includes data on prescriptions redeemed at community pharmacies by Danish residents. ${ }^{13,21}$ The National Health Service Register (NHSR) dates back to 1990 and includes data on health care services provided within the primary sector. ${ }^{21,22}$

\section{Psychiatric treatment}

Psychiatric treatment will be defined as at least one treatment redeemed/received by a patient. Data will be retrieved for each interval, and treatments redeemed/received by $<100$ patients will be excluded.

Pharmacological treatment: data on prescriptions redeemed from pharmacies by patients will be extracted from NPR. ${ }^{13}$ Data to be retrieved are Anatomical Therapeutic Chemical (ATC) codes. The following ATC codes will be retrieved at the $5^{\text {th }}$ ATC level: N03AX09 (lamotrigine), N03AX12 (gabapentin), N03AX16 (pregablin), N05**** (psycholeptics), N06A*** (antidepressants), N06C*** (psycholeptics and psychoanaleptics in combination) and N07B*** (drugs used in addictive disorders).

Non-pharmacological treatment: data on treatment received by patients in hospital settings will be extracted from DNPR. Data to be retrieved are SKS codes (Danish Health Care Classification System) at the highest level of specialization, albeit these will be manually clustered after data collection. ${ }^{15}$ The SKS codes to be retrieved are; BRKP*** (all treatments [***] with psychoeducation [BRKP]), BRS**** (all treatments [****] with conversation-based therapy [BRS]), BRT**** (all treatments [****] related to deprivation of liberty and other coercive psychiatric treatments [BRT]) and BRX**** (all $[* * * *]$ other interventions in relation to mental functions [BRX]). Data on treatment received from general and specialized practitioners will be extracted from NHSR, identified as the following SERVICE codes (Danish: SPECIALE koder $)^{22} ; 24 * * * *$ (all services [****] provided by a psychologist [24]), 26**** (all services [****] provided by children and adolescent psychiatry [26]), 63**** (all services [****] provided by a psychiatrist [63]) and 802448 (conversationbased therapy [2448] provided by a general practitioner [80]).

\section{Covariables}

Variables for characterization purposes will be: Diagnosis specifications: The ICD-10 code will be retrieved from the psychiatric domain of the DNPR and categorized into F32 (single episode) and F33 (recurrent). ${ }^{4}$ Additionally, severity will be categorized as mild (F3X.0), moderate (F3X.1), severe (F3X.2 and F3X.3) and unspecified (all other, e.g. F3X.9). ${ }^{4}$ Sex: Data on sex will be retrieved from the CRS. Age: the date of birth will be received from the CRS and age at index date grouped as; $<18$ years, $18-34$ years, 35-64 years and $>64$ years. Education: Data on education will be retrieved as the highest completed education at index date and categorized as: group 1 (primary, lower secondary), group 2 (upper secondary, short cycle), group 3 (Bachelor, Master, Doctoral) and group 4 (missing or not classified).

\section{Statistical analysis}

Latent class analysis

Psychiatric treatment profiles will be identified by latent class analysis (LCA). LCA is used to identify latent classes (i.e. groups) of individuals with homogenous characteristics or behavioural patterns, e.g. treatment patterns. ${ }^{23-26}$ The method will be used to model a finite number of groups at each of the six intervals, and these groups will be referred to as psychiatric treatment profiles.

Patients will be randomly assigned into two datasets to cross-validate the model fitting process. ${ }^{27}$ Model fitting is an iterative process that fits prespecified model solutions (i.e. the number of groups) to the data. The Bayesian Information Criterion (BIC) will be used to model selection together with a clinical judgement. ${ }^{23,27,28}$ The fitting process for each dataset will be initiated by a one-class model solution and continued by adding one class to the model solution until the BIC has increased twice. ${ }^{27-29}$ Once model solutions have been identified for each interval, a comparison of profiles across intervals will take place to assess whether the same profile appears in several intervals. Assignment of patients to profiles will be based on modal assignment, i.e. patients will be assigned to the profile in which they have the highest probability to belong according to the posterior probabilities. ${ }^{24,26}$ Trajectories will be established by cross-tabulating patients' profiles at different intervals. 


\section{Descriptive statistics}

For characterization purposes, descriptive statistics will be used. Median with interquartile range (IQR) will be used for continuous variables. For categorical variables, frequencies with percentages will be used.

Sample size calculation

The adequate sample size for LCA depends on two main aspects. Firstly, the effectiveness of the indicators to separate classes, and secondly, whether the size of the classes, once separated, is adequate to detect differences. ${ }^{23}$ Nevertheless, 300 subjects are suggested as a minimum cohort. ${ }^{28}$ Considering the prevalence of MDD and the eligibility period of five years (2011-2015), we expect that the cohort will exceed 30,000 subjects and that the number of patients of each profile will therefore be adequate.

\section{Statistical software}

The forthcoming study will access data from research servers at Statistics Denmark. ${ }^{13}$ Statistical Analysis System (SAS) Enterprise Guide $^{\circledR}$ (SCR_008567) will be available on the research servers from which data management will be led. Alternatively, the open-source software R-Studio (SCR_001905) can be used.

\section{Deliverable/data synthesis}

In the research article, the results will be presented in graphs, tables and diagrams. Two different graphs will be computed; plot constructs with BIC (y-axis) versus model solutions (x-axis), and plot constructs with the conditional probability (yaxis) versus psychiatric treatment (x-axis). ${ }^{27}$ These graphs will represent the model fitting and model selection process, respectively. The identified profiles, their prevalence and treatment patterns (conditional probabilities) will be presented in a table alongside profile-specific patient characteristics (Table 1). Likewise, the most frequent trajectories will be represented in a table (Table 2). Lastly, the most frequent trajectories will be depicted by a Sankey diagram in order to visualize the shifts between profiles and the magnitude of these shifts.

\section{Table 1. Example of table setup.}

\begin{tabular}{|c|c|c|c|c|}
\hline & Cohort & Profile 1 & $\ldots$ & Profile $X$ \\
\hline Frequency (n) & * & * & $\ldots$ & * \\
\hline \multicolumn{5}{|l|}{ Clinical factors } \\
\hline \multicolumn{5}{|c|}{ MDD distribution (\%) } \\
\hline$-F 32$ & * & * & $\ldots$ & * \\
\hline \multicolumn{5}{|l|}{ Severity (\%) } \\
\hline - Mild & * & * & $\ldots$ & * \\
\hline - Moderate & * & * & & * \\
\hline - Severe & * & * & & * \\
\hline - Unspecified & * & * & & * \\
\hline \multicolumn{5}{|c|}{ Sociodemographic factors } \\
\hline Age (Median, IQR) & * & * & $\ldots$ & * \\
\hline$-<18(\%)$ & * & * & & * \\
\hline$-18-34$ & * & * & & * \\
\hline - 35-64 (\%) & * & * & & * \\
\hline - >64 (\%) & * & * & & * \\
\hline \multicolumn{5}{|l|}{ Gender (\%) } \\
\hline - Female & * & * & $\ldots$ & * \\
\hline \multicolumn{5}{|c|}{ Level of education (\%) } \\
\hline - Group 1 & * & * & $\ldots$ & * \\
\hline - Group 2 & * & * & & * \\
\hline - Group 3 & * & * & & * \\
\hline - Group 4 & * & * & & * \\
\hline
\end{tabular}


Table 1. Continued

\begin{tabular}{|c|c|c|c|c|}
\hline & Cohort & Profile 1 & $\ldots$ & Profile X \\
\hline \multicolumn{5}{|c|}{ Profile characteristics } \\
\hline \multicolumn{5}{|c|}{ Class membership probabilities (Gamma) } \\
\hline - Int. 1 & * & * & $\ldots$ & * \\
\hline - Int. ... & * & * & & * \\
\hline - Int. 6 & * & * & & * \\
\hline \multicolumn{5}{|c|}{ Psychiatric treatments (rho) } \\
\hline - No. 1 & * & * & $\ldots$ & * \\
\hline - No.... & * & * & & * \\
\hline - No. X & * & * & & * \\
\hline
\end{tabular}

*Indicate where results will be presented.

MDD: major depressive disorder. Int.: interval. IQR: interquartile range.

Table 2. Example of table setup.

\begin{tabular}{|c|c|c|c|c|}
\hline & Cohort & Trajectory 1 & $\ldots$ & Trajectory $\mathrm{X}$ \\
\hline Frequency (n) & * & * & $\ldots$ & * \\
\hline \multicolumn{5}{|l|}{ Clinical factors } \\
\hline \multicolumn{5}{|c|}{ MDD distribution (\%) } \\
\hline$-F 32$ & * & * & $\ldots$ & * \\
\hline \multicolumn{5}{|l|}{ Severity (\%) } \\
\hline - Mild & * & * & $\ldots$ & * \\
\hline - Moderate & * & * & & * \\
\hline - Severe & * & * & & * \\
\hline - Unspecified & * & * & & * \\
\hline Age (Median, IQR) & * & * & $\ldots$ & * \\
\hline$-<18(\%)$ & * & * & & * \\
\hline - 18-34 (\%) & * & * & & * \\
\hline - 35-64 (\%) & * & * & & * \\
\hline - >64 (\%) & * & * & & * \\
\hline \multicolumn{5}{|c|}{ Sociodemographic factors } \\
\hline \multicolumn{5}{|c|}{ Gender (\%) } \\
\hline - Female & * & * & $\ldots$ & * \\
\hline \multicolumn{5}{|c|}{ Level of education (\%) } \\
\hline - Group 1 & * & * & $\ldots$ & * \\
\hline - Group 2 & * & * & & * \\
\hline - Group 3 & * & * & & * \\
\hline - Group 4 & * & * & & * \\
\hline \multicolumn{5}{|c|}{ Trajectory characteristics } \\
\hline Profiles (n) & NA & * & $\ldots$ & * \\
\hline - Profile 1 & NA & * & & * \\
\hline - Profile ... & NA & * & & * \\
\hline - Profile X & & & & \\
\hline
\end{tabular}

*Indicate where results will be presented.

MDD: major depressive disorder. IQR: interquartile range. NA: not applicable. 
Research ethics

The register-based study design does not require approval from an ethics committee according to Danish law. ${ }^{21}$ The Danish Data protection Agency has approved the study (reference number P-2020-88).

Dissemination plan

The results will be submitted for publication to an international peer-reviewed journal.

\section{Discussion}

\section{Limitations}

Time intervals are positioned relative to patients' index dates, thus intervals are patient-specific, meaning that the same interval is likely to occur at different time points for different patients and thereby be influenced by time-specific trends in treatment, legislation, guidelines, or new treatments. However, as the inclusion period only spans five years, we do not expect this to have a major impact on the results. Psychiatric treatment profiles are based on treatments redeemed/ retrieved within a four-month interval. Consequent, any shifts/discontinuation of treatment that may occur within these four months will not be represented by the psychiatric treatment profiles. Thus, the profiles could, misleadingly, be interpreted as polypharmacy or false treatment combinations. However, as many treatments are prescribed for three months at a time, we do not expect this to be an issue. In addition, we expect fewer treatment shifts in intervals further away from index time.

The ATC codes will be assessed at the $5^{\text {th }}$ level of the ATC system. At this level, the number of indicator variables (of the pharmacological treatment) is maximized. This might increase the complexity and limit the power of the information gained from the psychiatric treatment profiles. Nevertheless, there are clinical differences at the $5^{\text {th }}$ level of the ATC system (e.g. adverse effects), and an assessment at the $4^{\text {th }}$ level of the ATC system would mask clinical preferences. ${ }^{30}$

Psychiatric treatment that are redeemed/received by less than 100 patients will be excluded, i.e. treatment rarely redeemed/received by patients will not be included. This will lead treatment given to a minor subset of the study population to go undetected.

\section{Selection bias}

The universal coverage in Denmark minimizes the risk of selection bias. ${ }^{15}$ The overall validity of a single depression diagnosis (F32.0-3) identified in DNPR was found to be $72.8 \%$ for children and adolescents. ${ }^{31}$ The overall validity of the same diagnoses was found to be similar for adults (overall validity of $75.4 \%$ ). ${ }^{31,32}$ We expect the validity to be similar for a recurrent depression diagnosis (F33.0-3). The eligibility criteria will influence the study population composition which may introduce false homogeneity, e.g., the criterion of not having certain psychiatric diagnoses 15 years prior to index date can exclude patients with psychiatric comorbidity. This may skew the representativeness of the study population and thereby limit generalizability of the results.

\section{Information bias}

The mandatory and mainly electronic reporting of data into registers minimizes the overall risk of information bias. ${ }^{13,15}$ Pharmacological treatment data will exclusively be collected from the NPR. The register is considered complete and valid but still considered to have some drawbacks. ${ }^{13}$ The NPR lacks data on the indication of use, treatment duration, dosage, and adherence. The study, therefore, assumes that the pharmacological treatment is intended for MDD and consumed by the patient. ${ }^{13}$ Moreover, the NPR does not include pharmacological treatment received at hospitals; however, less than $1 \%$ of all antidepressants are given at the hospital and the majority of patients are treated by their general practitioner outside hospital settings. ${ }^{33,34}$ We do therefore not believe that lack of data on pharmacological treatment at hospitals will influence our results.

Non-pharmacological treatment data will be extracted from the DNPR and NHSR. Change in registration codes over time, varying registration practice and the lack of mutual exclusiveness can introduce information bias in data from the DNPR.$^{15}$ We believe that the same concerns are likely to influence data from the NHSR. Furthermore, the NHSR does not include treatment not covered by the Danish health care insurance (Danish: Sygesikringen), therefore data on patientfinanced treatment is not included.

Validation and quality control

All data management will be internally validated.

\section{Data availability}

No data are associated with this article. 
1. Ribeiro Â, Ribeiro JP, von Doellinger O: Depression and psychodynamic psychotherapy. Braz. J. Psychiatry. 2018; 40(1): 105-109.

PubMed Abstract | Publisher Full Text

2. The Danish Health Authority: The Burden of Disease in Denmark [Document]. 2015.

3. Kessler RC, Bromet EJ: The epidemiology of depression across cultures. Annu. Rev. Public Health. 2013; 34: 119-138. PubMed Abstract | Publisher Full Text

4. The World Health Organization: The ICD-10 Classification of Mental and Behavioural Disorders: Clinical descriptions and diagnostic guidelines [Document]

5. The Danish Health Authority: Reference program of unipolar depression in adults [Document]. 2007.

6. Dean J, Keshavan M: The neurobiology of depression: An integrated view. Asian J. Psychiatr. 2017 Jun; 27: 101-111. PubMed Abstract | Publisher Full Text

7. Kennis M, Gerritsen L, van Dalen M, et al.: Prospective biomarkers of major depressive disorder: a systematic review and metaanalysis. Mol. Psychiatry. 2020; 25(2): 321-338. PubMed Abstract | Publisher Full Text

8. The Danish Health Authority: Professional Guidelines for Psychologist Referral [Document]. 2012.

9. Menke A: Is the HPA Axis as Target for Depression Outdated, or Is There a New Hope?. Front. Psych. 2019 Feb 10; 10: 101. PubMed Abstract | Publisher Full Text

10. Manchia M, Pisanu C, Squassina A, et al.: Challenges and Future Prospects of Precision Medicine in Psychiatry. Pharmacogenomics Pers. Med. 2020; 13: 127-140. PubMed Abstract | Publisher Full Text

11. Gronemann $\mathrm{FH}$, Jorgensen $\mathrm{MB}$, Nordentoft $\mathrm{M}$, et al.: Sociodemographic and clinical risk factors of treatment-resistant depression: A Danish population-based cohort study. J. Affect. Disord. 2020 Jan 15; 261: 221-229. PubMed Abstract | Publisher Full Text

12. Keks N, Hope J, Keogh S: Switching and stopping antidepressants. Aust. Prescr. 2016 Jun; 39(3): 76-83. PubMed Abstract | Publisher Full Text

13. Pottegård A, Schmidt SAJ, Wallach-Kildemoes $\mathrm{H}$, et al.: Data Resource Profile: The Danish National Prescription Registry. Int. J. Epidemiol. 2016 Oct 27; 46: dyw213-dy798f. PubMed Abstract | Publisher Full Text

14. Schmidt M, Schmidt SAJ, Adelborg K, et al.: The Danish health care system and epidemiological research: from health care contacts to database records. CLEP. 2019 Jul; 11: 563-591. PubMed Abstract | Publisher Full Text

15. Schmidt M, Schmidt SAJ, Sandegaard JL, et al.: The Danish National Patient Registry: a review of content, data quality, and research potential. CLEP. 2015 Nov; 449 Publisher Full Text

16. Schmidt M, Pedersen L, Sørensen HT: The Danish Civil Registration System as a tool in epidemiology. Eur. J. Epidemiol. 2014 Aug; 29(8): 541-549. PubMed Abstract | Publisher Full Text

17. Erlangsen A, Fedyszyn I: Danish nationwide registers for public health and health-related research. Scand. J. Public Health. 2015 Jun; 43(4): 333-339.

PubMed Abstract | Publisher Full Text

18. Pedersen CB: The Danish Civil Registration System. Scand. J. Public Health. 2011 Jul 1; 39(7_suppl): 22-25 Publisher Full Text
19. Lynge E, Sandegaard JL, Rebolj M: The Danish National Patient Register. Scand. J. Public Health. 2011 Jul 1; 39(7_suppl): 30-33. Publisher Full Text

20. Mors O, Perto GP, Mortensen PB: The Danish Psychiatric Central Research Register. Scand. J. Public Health. 2011 Jul; 39(7_suppl): 54-57.

Publisher Full Text

21. Thygesen LC, Daasnes C, Thaulow I, et al.: Introduction to Danish (nationwide) registers on health and social issues: Structure, access, legislation, and archiving. Scand.J. Public Health. $2011 \mathrm{Jul} 1$ 39(7_suppl): 12-16.

PubMed Abstract | Publisher Full Text

22. Sahl Andersen J, De Fine ON, Krasnik A: The Danish National Health Service Register. Scand. J. Public Health. 2011 Jul 1; 39(7_suppl): 34-37. Publisher Full Text

23. Sinha P, Calfee CS, Delucchi KL: Practitioner's Guide to Latent Class Analysis: Methodological Considerations and Common Pitfalls. Crit. Care Med. 2021 Jan; 49(1): e63-e79. PubMed Abstract | Publisher Full Text

24. Lanza ST, Cooper BR: Latent Class Analysis for Developmental Research. Child Dev. Perspect. 2016 Mar; 10(1): 59-64. PubMed Abstract | Publisher Full Text

25. Collins LM, Lanza ST: "General Introduction". Latent Class and Latent Transition Analysis: With Applications in the Social, Behavioral, and Health Sciences.1st ed. John Wiley \& Sons, Inc.; 2010; 3-22.

26. Kongsted A, Nielsen AM: Latent Class Analysis in health research. J. Physiother. 2017 Jan 1; 63(1): 55-58. Publisher Full Text

27. Collins LM, Lanza ST: Parameters estimation and mode selection. Latent Class and Latent Transition Analysis: With Applications in the Social, Behavioral, and Health Sciences.1st ed. John Wiley \& Sons, Inc.; 2010. 77-110.

28. Weller BE, Bowen NK, Faubert SJ: Latent Class Analysis: A Guide to Best Practice. J. Black Psychol. 2020 May 1; 46(4): 287-311. Publisher Full Text

29. Nylund KL, Asparouhov T, Muthén BO: Deciding on the Number of Classes in Latent Class Analysis and Growth Mixture Modeling: A Monte Carlo Simulation Study. Struct. Equ. Model. Multidiscip. J. 2007 Oct 23; 14(4): 535-569. Publisher Full Text

30. Gerald G, Kylie T, Hill S, et al.: How Should Primary Care Doctors Select Which Antidepressants to Administer?. Curr. Psychiatry Rep. 2012 Aug; 14(4): 360-369. Publisher Full Text

31. Frederiksen $\mathrm{LH}$, Bilenberg $\mathrm{N}$, Andersen L, et al.: The validity of child and adolescent depression diagnoses in the Danish psychiatric central research register. Acta Psychiatr. Scand. 2021; 143(3): 264-274.

PubMed Abstract | Publisher Full Text

32. Bock C, Bukh JD, Vinberg M, et al.: Validity of the diagnosis of a single depressive episode in a case register. Clin. Pract. Epidemiol. Ment. Health. 2009 Feb; 5(5): 4 Publisher Full Text

33. Council for the Use of Expensive Hospital medicines: Pharmacological treatment of unipolar depression in adults [Document]. 2015.

34. The Danish Health Authority: National Clinical Guideline for the Non-pharmacological treatment of Unipolar Depression [Document]. 2016. 
The benefits of publishing with F1000Research:

- Your article is published within days, with no editorial bias

- You can publish traditional articles, null/negative results, case reports, data notes and more

- The peer review process is transparent and collaborative

- Your article is indexed in PubMed after passing peer review

- Dedicated customer support at every stage

For pre-submission enquiries, contact research@f1000.com 